disorders in del(5q) myelodysplastic syndromes. Haematologica 2013; 98 : 1856-1864.

6 Pellagatti A, Boultwood J. The molecular pathogenesis of the myelodysplastic syndromes. Eur J Haematol 2015; 95; 3-15.

7 Bejar R, Stevenson K, Abdel-Wahab O, Galili N, Nilsson B, Garcia-Manero G et al. Clinical effect of point mutations in myelodysplastic syndromes. $N$ Engl J Med 2011; 364: 2496-2506.

8 Malcovati L, Papaemmanuil E, Bowen DT, Boultwood J, Della Porta MG, Pascutto $C$ et al. Clinical significance of SF3B1 mutations in myelodysplastic syndromes and myelodysplastic/myeloproliferative neoplasms. Blood 2011; 118: 6239-6246.

9 Mian SA, Smith AE, Kulasekararaj AG, Kizilors A, Mohamedali AM, Lea NC et al. Spliceosome mutations exhibit specific associations with epigenetic modifiers and proto-oncogenes mutated in myelodysplastic syndrome. Haematologica 2013; 98: 1058-1066.

10 Genovese G, Kahler AK, Handsaker RE, Lindberg J, Rose SA, Bakhoum SF et al. Clonal hematopoiesis and blood-cancer risk inferred from blood DNA sequence. N Engl J Med 2014; 371: 2477-2487.

11 Jaiswal S, Fontanillas P, Flannick J, Manning A, Grauman PV, Mar BG et al. Age-related clonal hematopoiesis associated with adverse outcomes. $N$ Engl J Med 2014; 371: 2488-2498.
12 Cazzola M, Della Porta MG, Malcovati L. The genetic basis of myelodysplasia and its clinical relevance. Blood 2013; 122: 4021-4034.

13 Abdel-Wahab O, Adli M, LaFave LM, Gao J, Hricik T, Shih AH et al. ASXL1 mutations promote myeloid transformation through loss of PRC2-mediated gene repression. Cancer Cell 2012; 22: 180-193.

14 Walter MJ, Shen D, Shao J, Ding L, White BS, Kandoth C et al. Clonal diversity of recurrently mutated genes in myelodysplastic syndromes. Leukemia 2013; 27: 1275-1282.

$15 \mathrm{Xu} \mathrm{L,} \mathrm{Gu} \mathrm{ZH,} \mathrm{Li} \mathrm{Y,} \mathrm{Zhang} \mathrm{JL,} \mathrm{Chang} \mathrm{CK,} \mathrm{Pan} \mathrm{CM} \mathrm{et} \mathrm{al.} \mathrm{Genomic} \mathrm{landscape}$ of $\mathrm{CD} 34+$ hematopoietic cells in myelodysplastic syndrome and gene mutation profiles as prognostic markers. Proc Natl Acad Sci USA 2014; 111: 8589-8594.

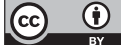

This work is licensed under a Creative Commons Attribution 4.0 International License. The images or other third party material in this article are included in the article's Creative Commons license, unless indicated otherwise in the credit line; if the material is not included under the Creative Commons license, users will need to obtain permission from the license holder to reproduce the material. To view a copy of this license, visit http://creativecommons.org/licenses/ by/4.0/

Supplementary Information accompanies this paper on the Leukemia website (http://www.nature.com/leu)

\title{
OPEN
}

\section{The leukemia-associated RUNX1/ETO oncoprotein confers a mutator phenotype}

Leukemia (2016) 30, 250-253; doi:10.1038/leu.2015.133

$t(8 ; 21)$ is a frequent chromosomal translocation in acute myeloid leukemia (AML) and is also reported in lymphoid and biphenotypic acute leukemia. ${ }^{1,2} \mathrm{t}(8 ; 21)$ fuses the $R U N X 1$ gene $(A M L 1)$ on chromosome 21 to the ETO gene (RUNX1T1) on chromosome 8, encoding the RUNX1/ETO chimeric transcription factor that represses expression of RUNX1 target genes, promoting selfrenewal and blocking myeloid differentiation. ${ }^{3-6} \mathrm{t}(8 ; 21)$ is insufficient for leukemogenesis and additional co-operating mutations are required for transformation, ${ }^{7}$ including point mutations that activate and/or over express c-KIT. ${ }^{8}$ The mechanisms driving the acquisition of co-operating mutations remain unclear, although there is evidence that initiating lesions such as RUNX1/ETO may promote mutagenesis. ${ }^{9,10}$ For example, ectopic expression of RUNX1/ETO downregulates several DNA-repair proteins (BRCA2, OGG1 and ATM) and increases the level of phosphorylated TP53 and $\mathrm{YH} 2 \mathrm{AX}$, indicating elevated DNA damage and a possible pro-mutagenic phenotype. ${ }^{10,11}$

To test whether expression of RUNX1/ETO is sufficient to increase genomic instability and mutagenesis, we expressed RUNX1/ETO in the non-transformed TK6 lymphoblastoid cell line and measured the acquisition of mutations at the PIGA reporter gene. Cells were transduced with lentivirus carrying either enhanced green fluorescent protein (EGFP) and the RUNX1/ETO fusion or EGFP alone (vector control) (Supplementary Figure 1A; Supplementary Methods) and clones expressing both RUNX1/ETO mRNA and protein were generated (Supplementary Figures 1B and C). For the purposes of measuring RUNX1/ETO-induced mutagenesis, we analysed independent cell clones expressing at least $40 \%$ of the fusion transcript found in the Kasumi-1 and SKNO-1 t(8;21)positive cell lines (Supplementary Figure 1B). Monitoring of
RUNX1/ETO-positive clones revealed that expression of the fusion was stable over long culture periods ( $>12$ weeks). Independent vector control clones that expressed equivalent levels of EGFP to RUNX1/ETO-positive cells were used as fusionprotein negative controls (Supplementary Figure 1D).

PIGA encodes a protein essential for production of the glycosylphosphatidylinositol anchor, mediating anchoring of proteins including CD55 and CD59 to the cell membrane. ${ }^{12}$ Somatic mutations of PIGA are growth neutral and can be determined using flow cytometry analysis measuring CD55 and CD59 expression (Supplementary Figure 2; Supplementary Methods). Using this reporter gene system we determined the mutation frequency (Mf) in several independent RUNX1/ETOexpressing and vector control clones after continuous culture for 8-10 weeks after initial cloning. All clones had a majority population that was positive for CD55 and CD59, confirming that the founding cell was PIGA wild-type (WT). The mean PIGA Mf in RUNX1/ETO clones $\left(7.50 \times 10^{-4}\right)$ was five times higher than in vector control clones $\left(1.45 \times 10^{-4}\right)$ (Figure $1 \mathrm{a}$, Supplementary Figure $3 \mathrm{~A})(P=0.032)$, with the exception of a single clone that had low expression of the fusion transcript (equivalent to $40 \%$ of that in Kasumi-1) (Supplementary Figures 1B and 3A).

The frequency of cells with a mutation $(\mathrm{M} f)$ at a specific gene is determined by the mutation rate per cell division for that gene $(\mu)$ and the number of cell divisions (d). Expression of the RUNX1/ETO fusion had a modest but nonsignificant negative effect on cell proliferation (Figure 1b, Supplementary Figure 3B, $P=0.812$ ), confirming that the increased $M f$ in RUNX1/ETO fusion-positive cells was not due to elevated proliferation.

Despite being derived from single cells, EGFP expression in RUNX1/ETO clones was normally distributed in all cell populations (Figure 1c, Supplementary Figure 4), indicating a natural drift in EGFP post cloning. Our data suggest that RUNX1/ETO confers a 
a

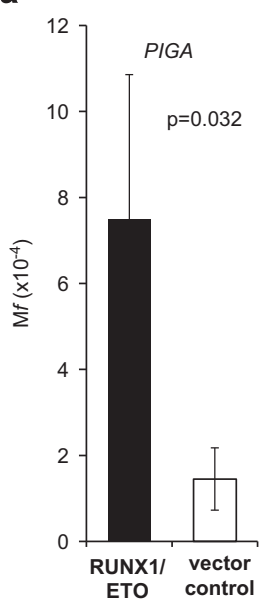

b

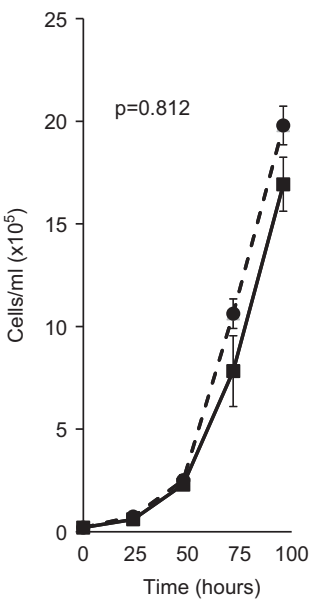

C
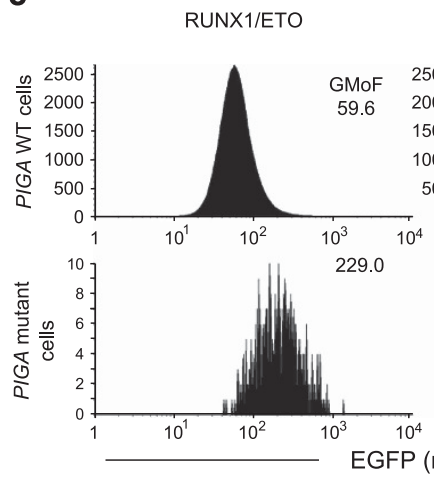
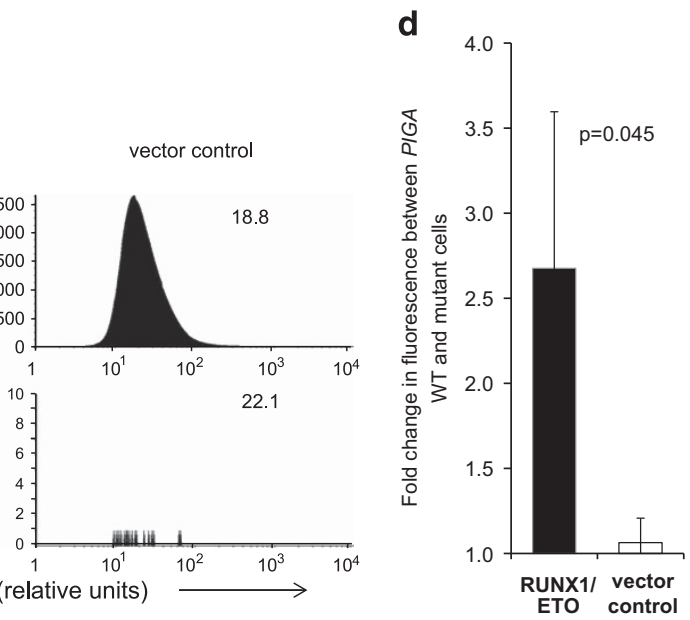

Figure 1. Expression of RUNX1/ETO increases spontaneous Mf. (a) RUNX1/ETO clones (black bar) and vector control clones (white bar) were assayed for Mf at the PIGA gene. RUNX1/ETO and vector control clones were cultured post cloning for 8-10 weeks before assessment of PIGA Mf. $\mathrm{M} f$ was calculated by quantifying the number of PIGA-negative cells, which had passed all gating steps described in Supplementary Figure 2 and dividing this by the total number of cells that had passed all gating steps described in Supplementary Figure 2. M $f$ values are the mean of five RUNX1/ETO clones and four vector control clones. RUNX1/ETO clones showed a significant increase $(P=0.032)$ in $P I G A$ M $f$ compared with vector control clones. (b) Growth curves of RUNX1/ETO (solid line) and vector control cells (dashed line). Cells were seeded at $2 \times 10^{4} / \mathrm{ml}$ and cell proliferation in six RUNX1/ETO clones and five vector control clones was measured every $24 \mathrm{~h}$ for 4 days. Mean cells/ml value of all clones is displayed and error bars represent the s.d. No significant difference was observed between the proliferation of RUNX1/ETO clones and vector control clones ( $P=0.812$; ANOVA). (c) Example flow cytogram plots showing a higher EGFP level in PIGA mutant cells (bottom left panel) compared with WT PIGA cells (top left panel) from a single RUNX1/ETO clone. In contrast, differential EGFP expression between PIGA mutant and WT cells was not observed in vector control cells from a single clone (right panels) (further examples are shown in Supplementary Figure 4). Numbers represent EGFP geometric mean of fluorescence (GMoF). (d) EGFP levels in PIGA mutant and WT cells. EGFP levels (a surrogate for RUNX1/ETO) in PIGA mutant and WT cells were measured in RUNX1/ETO clones (black bars) and vector control clones (white bars) and represented as fold change of EGFP fluorescence between PIGA WT and mutant cells. Histogram shows the mean from five RUNX1/ETO clones and four vector control clones. Error bars represent the standard deviation. RUNX1/ETO PIGA mutants had significantly higher EGFP fluorescence than PIGA WT RUNX1/ETO cells from the same population, whereas no significant difference was observed between vector control PIGA mutant and WT cells $(P=0.045$; one tailed unpaired Student's $t$-test using fold change in fluorescence between PIGA WT and mutant cells and comparing RUNX1/ETO clones and vector control clones).

mutator phenotype in a protein-level-dependent fashion. By using EGFP as a surrogate for RUNX1/ETO we predicted that PIGA-mutant cells would have higher EGFP expression compared with nonmutant cells from the same population. Consistent with this hypothesis, the EGFP geometric mean of fluorescence (GMoF) of PIGA mutant cells derived from RUNX1/ETO cell clones was significantly higher than in non-mutants derived from the same starting population (Figures $1 \mathrm{c}$ and d, Supplementary Figure 4, Supplementary Table 1). The only RUNX1/ETO clone that did not show a difference between EGFP GMoF for PIGA WT and mutant cells was that with the lowest RUNX1/ETO expression (clone RE4), which also had no increase in spontaneous PIGA Mf (Supplementary Figure $3 \mathrm{~A}$ ). For vector control clones, the EGFP GMoF was not significantly different between PIGA mutant and WT cells (Figure 1d, Supplementary Figure 4, Supplementary Table 1). These data demonstrate that expression of the RUNX1/ETO fusion oncoprotein confers a mutator phenotype when the transcript is at levels equivalent to at least $60 \%$ of that in $t(8 ; 21)$-positive cell lines.

We next investigated whether RUNX1/ETO transduced cells were particularly sensitive to mutagenesis following exposure to genotoxic anticancer therapies. Fusion protein-positive and vector control clones were exposed to sub-cytotoxic doses of either doxorubicin (100 $\mathrm{nm}$ for $4 \mathrm{~h}$ ) or ionising radiation ( $3 \mathrm{~Gy}$ ), and $\mathrm{Mf}$ was measured 2 weeks after treatment. The frequency of mutations attributable to doxorubicin or radiation (treatmentinduced $M f$ ) was calculated by subtracting the Mf in mock-treated cells from the $\mathrm{M} f$ in doxorubicin- or radiation-treated cells. The mean treatment-induced PIGA Mf was strongly inceased in RUNX1/ETO cell clones compared with vector control clones following either doxorubicin (mean $\mathrm{Mf}=6.54 \times 10^{-4}$ vs $0.208 \times 10^{-4}$, $P=0.09$ ) or radiation treatment (mean $M f=5.92 \times 10^{-4}$ vs
$0.209 \times 10^{-4}, P=0.008$ ) (Figure 2a, Supplementary Figure 5). As an additional independent measure for treatment-induced $M f$ we assayed RUNX1/ETO and vector control clones at a second reporter gene, thymidine kinase (TK). The mean treatmentinduced $T K M f$ was also significantly higher in RUNX1/ETO cell clones compared with vector control clones following either doxorubicin (mean $\mathrm{Mf}=3.53 \times 10^{-6}$ vs $0.432 \times 10^{-6}, P=0.014$ ) or radiation treatment (mean $\mathrm{Mf}=6.43 \times 10^{-6}$ vs $-0.145 \times 10^{-6}$, $P=0.002$ ) (Figure 2a, Supplementary Figure 5 ). The only RUNX1/ ETO clone that did not show increased sensitivity to doxorubicin or radiation-induced mutation was clone RE4 with low RUNX1/ETO expression (Supplementary Figure 5). For both PIGA and TK, the Mf in vector control clones following doxorubicin or radiation treatment was not significantly different from mock-treated cells (Figure 2a, Supplementary Figure 5), suggesting that, unlike RUNX1/ETO-expressing cells, vector control cells were proficient at repairing treatment-induced DNA damage.

We next investigated whether EGFP expression differed in treatment-induced PIGA mutant cells compared with non-mutant cells derived from the same RUNX1/ETO clonal population. The EGFP GMoF of PIGA mutant cells was significantly higher than in non-mutant cells following treatment with doxorubicin $(P=0.03)$ or ionising radiation $(P=0.02)$ (Figures $2 \mathrm{~b}$ and $\mathrm{C}$, Supplementary Figure 6). In contrast, the EGFP GMoF was not significantly different between PIGA mutant and WT cells derived from vector control populations following treatment (Figures $2 \mathrm{~b}$ and $\mathrm{c}$ ).

These data demonstrate that high levels of RUNX1/ETO sensitise cells to acquisition of mutations following treatment with DNA damage-inducing agents, suggesting that fusion-protein-expressing cells may be compromised in DNA repair. This result is consistent 
a
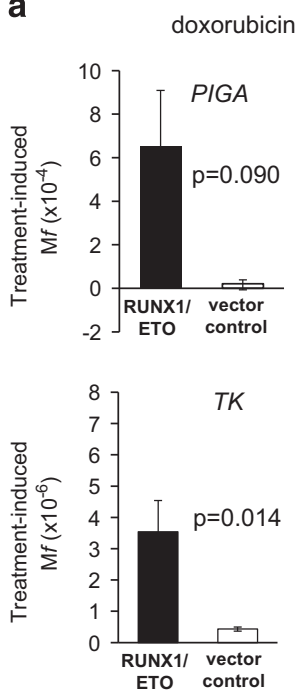

b doxorubicin
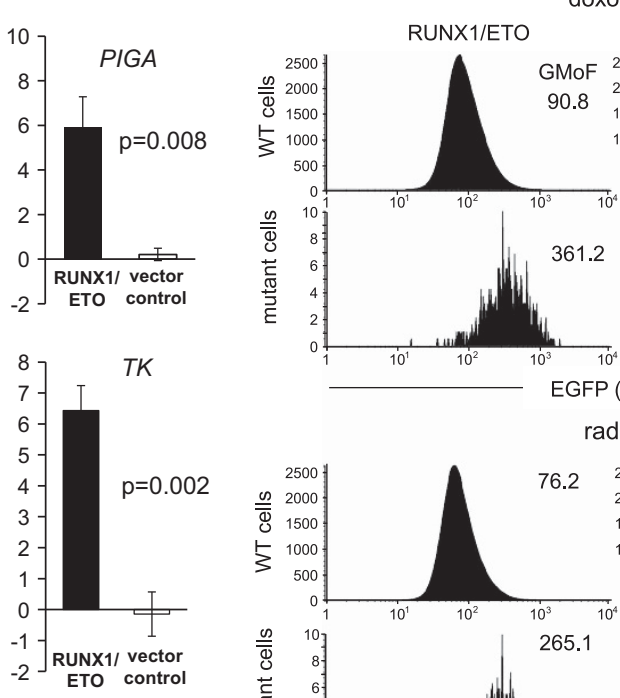
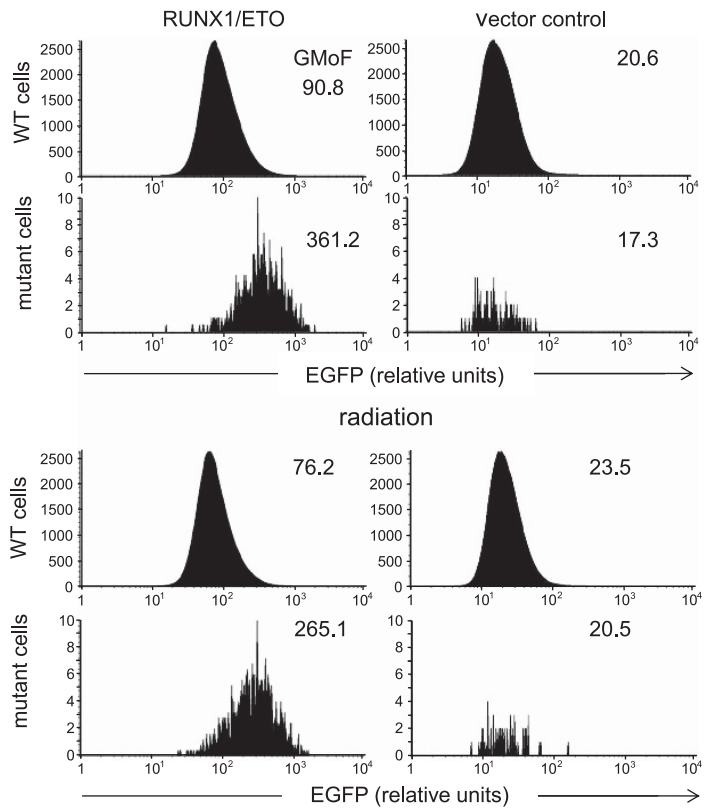

c

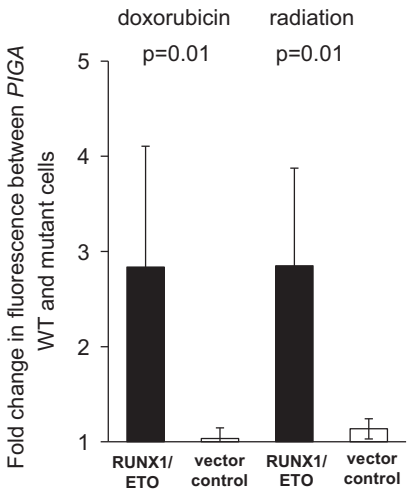

Figure 2. Expression of RUNX1/ETO increases Mf after dosing with doxorubicin or ionising radiation. (a) RUNX1/ETO clones (black bars) and vector control clones (white bars) were cultured for 3 weeks post cloning before treating with doxorubicin, radiation or mock, with an additional 2 weeks for phenotype development before assaying for Mf at the PIGA (top panels) and TK (bottom panels) genes. The frequency of mutations attributable to doxorubicin or radiation (treatment-induced $\mathrm{M} f$ ) was calculated by subtracting the $\mathrm{M} f$ in mock-treated cells from the $\mathrm{M} f$ in doxorubicin or radiation-treated cells. Treatment-induced $\mathrm{M} f$ values displayed are the mean of five RUNX1/ETO clones and four vector control clones for PIGA and three RUNX1/ETO clones and four vector control clones for TK. RUNX1/ETO clones showed a significant increase in Mf at PIGA ( $P=0.008$; unpaired Student's $t$-test, two tailed) and TK $(P=0.002)$ after radiation treatment. RUNX1/ETO clones showed a significant increase in $\mathrm{M} f$ at $T K$ after doxorubicin treatment $(P=0.014)$, and a non significant increase in M $f$ at $P I G A(P=0.09)$. (b) Example flow cytogram plots showing a higher EGFP level in PIGA mutant cells (bottom left panel for doxorubicin or radiation-treated cells) compared with WT PIGA cells (top left panels) from a single RUNX1/ETO clone. In contrast, differential EGFP expression between PIGA mutant and WT cells were not observed in vector control cells from a single clone after either doxorubicin or radiation treatment (right panels) (further examples are shown in Supplementary Figure 6). Numbers represent EGFP GMoF. (c) EGFP levels in PIGA mutant and WT cells after treatment with doxorubicin or ionising radiation. EGFP levels (a surrogate for RUNX1/ETO) in PIGA mutant and WT cells were measured in RUNX1/ETO clones (black bars) and vector control clones (white bars) and represented as fold change of EGFP fluorescence between PIGA WT and mutant cells. Histogram shows the mean from five RUNX1/ETO clones and four vector control clones. Error bars represent the standard deviation. RUNX1/ ETO PIGA mutants had a significantly higher EGFP fluorescence than PIGA WT RUNX1/ETO cells from the same population after treatment with doxorubicin or radiation, whereas no significant difference was observed between vector control PIGA mutant and WT cells $(P=0.01$ for doxorubicin and $P=0.01$ for radiation; one tailed unpaired Student's $t$-test using fold change in fluorescence between PIGA WT and mutant cells and comparing RUNX1/ETO clones and vector control clones).

with previously published reports implicating compromised OGG1 DNA glycosylase activity in $\mathrm{t}(8 ; 21)$ AML. ${ }^{11,13}$ OGG1 functions in base excision repair and initiates repair of oxidised lesions, including 8-hydroxy-2'-deoxyguanosine. We therefore investigated OGG1 expression in 18 independent RUNX1/ETO cell clones and observed that transcript levels were inversely proportional to the expression of the fusion gene (Supplementary Figure 7A). Likewise, OGG1 protein levels were consistently lower in RUNX1/ETO-positive clones compared with vector control clones (Supplementary Figure 7B). We next performed siRNA-mediated depletion of RUNX1/ETO in Kasumi-1, as previously described. ${ }^{5}$ Two serial electroporations with either RUNX1/ETO or control scrambled siRNA were carried out and OGG1 transcript and protein levels were assessed $72 \mathrm{~h}$ after each electroporation. RUNX1/ETO depletion increased OGG1 transcript levels by fourfold and sixfold on days 3 and 6, respectively (Supplementary Figure 7C) with a concomitant increase in OGG1 protein (Supplementary Figures $7 C$ and D). To confirm OGG1 as a RUNX1/ETO target gene we performed ChIP and RNA sequencing after siRNA-mediated RUNX1/ETO depletion. A RUNX1/ETO CHiPseq peak was observed at the OGG1 promoter in control-transfected Kasumi-1 cells, which was lost following siRNA-mediated fusion protein depletion (Supplementary Figure 7E) and was co-localised within a DNasel hypersensitivity site, as previously reported. ${ }^{6}$
We also confirmed the increase in OGG1 expression after RUNX1/ ETO depletion by RNAseq (Supplementary Figure 7E). Taken together, these data confirm that the RUNX1/ETO fusion protein binds the OGG1 promoter and negatively regulates transcription, suggesting a plausible mechanism by which this common fusion oncoprotein drives mutagenesis. In order to investigate this further, we interrogated whole-genome and exome sequencing data from The Cancer Genome Atlas Project. ${ }^{14}$ Data were available from 193 AML cases, which included information on 21386 somatic base substitution mutations, including $G>T$ transversions, the predominant mutation that arises following translesion synthesis of 8-hydroxy-2'-deoxyguanosine and which accumulates following knockdown of OGG1 in experimental systems. ${ }^{15}$ When stratified by cytogenetic subgroup, $16.13 \%$ of all mutations in $\mathrm{t}(8 ; 21)$ AML were $\mathrm{G}>\mathrm{T}$ transversions $(16.13 \%, n=7$ cases, Supplementary Table 2), which was higher than any other cytogenetic subgroup and all other AML cases combined (12.14\%, $n=186$ cases), although this did not reach statistical significance ( $P=0.31$, Yates chi-square). These data are consistent with loss of OGG1 contributing to the mutator phenotype in RUNX1/ETO cells by predisposing to G:C $>$ T:A transversions.

By using mutation assays using two independent assays, we have shown that RUNX1/ETO predisposes to the acquisition of 
mutations, both spontaneously and particularly after treatment with genotoxic agents. We also present evidence that the strength of the mutator phenotype is related to the expression level of the fusion protein. As with all types of AML, relapse is a major cause of mortality in $\mathrm{t}(8 ; 21) \mathrm{AML}$. If remission-induction chemotherapy fails to eliminate all the RUNX1/ETO-positive cells, our data suggest that surviving cells are predisposed to mutations that could drive relapse. One consideration for chemotherapy should therefore be to limit mutation of surviving leukemic/preleukemic cells. As such, we hypothesise that targeting the mutator phenotype associated with expression of the RUNX1/ETO fusion gene could impede the acquisition of co-operating mutations required for disease relapse. Our data demonstrate the need to develop novel therapeutic strategies that avoid increasing the mutation burden of AML cells.

\section{CONFLICT OF INTEREST}

The authors declare no conflict of interest.

\section{ACKNOWLEDGEMENTS}

This work was supported by grants from Leukaemia and Lymphoma Research (\#13044 to JMA and \#10033, \#12055 to $\mathrm{OH}$ ) and the JGW Patterson Foundation (to JMA and $\mathrm{OH}$ ). VJF designed and performed research, analysed data and wrote the manuscript. MHN NM-S, AKB, AP, SEF, HM and SAA performed research. $\mathrm{CB}$ and $\mathrm{OH}$ designed research and analysed data. JMA conceived the project, designed research, analysed data and wrote the manuscript. All authors approved the final version of the manuscript.

VJ Forster ${ }^{1}$, MH Nahari ${ }^{1}$, N Martinez-Soria ${ }^{1}$, AK Bradburn ${ }^{1}$, A Ptasinska ${ }^{2}$, SA Assi ${ }^{2}$, SE Fordham ${ }^{1}, \mathrm{H} \mathrm{McNeil}^{1}, \mathrm{C} \mathrm{Bonifer}^{2}$ O Heidenreich ${ }^{1}$ and JM Allan ${ }^{1}$

${ }^{1}$ Northern Institute for Cancer Research, Newcastle Cancer Centre, Newcastle University, Newcastle-upon-Tyne, UK and

${ }^{2}$ School of Cancer Sciences, Institute of Biomedical Research, College of Medical and Dental Sciences, University of Birmingham, Birmingham, UK

E-mail: olaf.heidenreich@newcastle.ac.uk or james.allan@newcastle.ac.uk

\section{REFERENCES}

1 Wang HY, Tirado CA. t(8;21)(q22;q22) Translocation involving AML1 and ETO in B lymphoblastic leukemia. Hum Pathol 2010; 41: 286-292.

2 He G, Wu D, Sun A, Xue Y, Jin Z, Qiu H et al. B-Lymphoid and myeloid lineages biphenotypic acute leukemia with $\mathrm{t}(8 ; 21)(\mathrm{q} 22 ; \mathrm{q} 22)$. Int J Hematol 2008; 87: 132-136.
3 Peterson LF, Zhang DE. The 8;21 translocation in leukemogenesis. Oncogene 2004; 23: 4255-4262.

4 Mulloy JC, Cammenga J, MacKenzie KL, Berguido FJ, Moore MA, Nimer SD. The AML1-ETO fusion protein promotes the expansion of human hematopoietic stem cells. Blood 2002; 99: 15-23.

5 Martinez Soria N, Tussiwand R, Ziegler P, Manz MG, Heidenreich O. Transient depletion of RUNX1/RUNX1T1 by RNA interference delays tumour formation in vivo. Leukemia 2009; 23: 188-190.

6 Ptasinska A, Assi SA, Martinez-Soria N, Imperato MR, Piper J, Cauchy P et al. Identification of a dynamic core transcriptional network in $t(8 ; 21)$ AML that regulates differentiation block and self-renewal. Cell Rep 2014; 8: 1974-1988.

7 Yuan $\mathrm{Y}$, Zhou L, Miyamoto $\mathrm{T}$, Iwasaki $\mathrm{H}$, Harakawa $\mathrm{N}$, Hetherington $\mathrm{CJ}$ et al. AML1-ETO expression is directly involved in the development of acute myeloid leukemia in the presence of additional mutations. Proc Natl Acad Sci USA 2001; 98: 10398-10403.

8 Wang YY, Zhou GB, Yin T, Chen B, Shi JY, Liang WX et al. AML1-ETO and C-KIT mutation/overexpression in $\mathrm{t}(8 ; 21)$ leukemia: implication in stepwise leukemogenesis and response to Gleevec. Proc Natl Acad. SC USA 2005; 102: 1104-1109.

9 Araten DJ, Krejci O, Ditata K, Wunderlich M, Sanders KJ, Zamecheck L et al. The rate of spontaneous mutations in human myeloid cells. Mutat Res 2013; 749: 49-57.

10 Krejci O, Wunderlich M, Geiger H, Chou FS, Schleimer D, Jansen $M$ et al. p53 signaling in response to increased DNA damage sensitizes AML1-ETO cells to stress-induced death. Blood 2008; 111: 2190-2199.

11 Alcalay M, Meani N, Gelmetti V, Fantozzi A, Fagioli M, Orleth A et al. Acute myeloid leukemia fusion proteins deregulate genes involved in stem cell maintenance and DNA repair. J Clin Investig 2003; 112: 1751-1761.

12 Chen R, Knez JJ, Merrick WC, Medof ME. Comparative efficiencies of C-terminal signals of native glycophosphatidylinositol (GPI)-anchored proproteins in conferring GPI-anchoring. J Cell Biochem 2001; 84: 68-83.

13 Liddiard K, Hills R, Burnett AK, Darley RL, Tonks A. OGG1 is a novel prognostic indicator in acute myeloid leukaemia. Oncogene 2010; 29: 2005-2012.

14 The Cancer Genome Atlas Research Network. Genomic and epigenomic landscapes of adult de novo acute myeloid leukemia. N Engl J Med 2013; 368: 2059-2074.

15 Suzuki T, Harashima $H$, Kamiya $H$. Effects of base excision repair proteins on mutagenesis by 8-oxo-7,8-dihydroguanine (8-hydroxyguanine) paired with cytosine and adenine. DNA Repair 2010; 9: 542-550.

\section{IL1RAP expression as a measure of leukemic stem cell burden at diagnosis of chronic myeloid leukemia predicts therapy outcome}

Leukemia (2016) 30, 253-257; doi:10.1038/leu.2015.135

Chronic myeloid leukemia (CML) is characterized by the $B C R / A B L 1$ fusion gene formed by a $\mathrm{t}(9 ; 22)$ chromosomal translocation. The $t(9 ; 22)$ is thought to arise in a hematopoietic stem cell creating a hierarchically arranged leukemia with a subset of cells in the $\mathrm{CD} 34^{+} \mathrm{CD} 38^{\text {low }}$ fraction comprising the primitive leukemia initiating cells, also termed leukemic stem cells. ${ }^{1,2}$ The treatment of CML was greatly improved with the introduction of tyrosine kinase inhibitors more than a decade ago, but not all patients respond optimally to the initial treatment and even after obtaining a deep molecular remission, discontinuation of treatment is often followed by a relapse. ${ }^{3-5}$

The leukemic stem cell (LSC) burden of CML patients at diagnosis could potentially provide an important disease variable 